\title{
Aplicativos móveis de saúde para redução do comportamento sedentário
}

\section{Mobile health applications to reduce sedentary behavior \\ Aplicaciones móviles de salud para reducir la conducta sedentaria}

(iD) Deisy Terumi Ueno

Universidade Estadual Paulista Júlio de Mesquita Filho, Rio Claro, São Paulo, Brasil. E-mail: terumiueno@uol.com.br

iD Angélica Bonolo

Universidade Estadual Paulista Júlio de Mesquita Filho, Rio Claro, São Paulo, Brasil. E-mail: angelicabonolo@gmail.com

iD@ Jean Augusto Coelho Guimarães Universidade Estadual Paulista Júlio de Mesquita Filho, Rio Claro, São Paulo, Brasil. E-mail: jean13ef@gmail.com

iD) Ana Elisa Messetti Christofoletti Universidade Estadual Paulista Júlio de Mesquita Filho, Rio Claro, São Paulo, Brasil. E-mail: anaelisamchr@gmail.com

Priscila Missaki Nakamura Instituto Federal de Educação, Ciências e Tecnologias - Sul de Minas Gerais (IFSULDEMINAS), Muzambinho, Minas Gerais, Brasil. E-mail: missaki.naka@gmail.com

iD Eduardo Kokubun Universidade Estadual Paulista Júlio de Mesquita Filho, Rio Claro, São Paulo, Brasil. E-mail: ekokubun@gmail.com

Resumo: O objetivo deste estudo foi identificar e agrupar informações de aplicativos móveis voltados para a redução do comportamento sedentário (CS) existentes nas plataformas Google Play e iTunes. O levantamento ocorreu em agosto de 2020, utilizando-se os termos relacionados 
ao CS. Foram encontrados 196 aplicativos, sendo a maioria disponível apenas em língua inglesa e gratuitos. Em geral, os aplicativos podem ser divididos em quatro categorias: a) alertas/lembretes para quebra do CS; b) Restrição de aplicativos e bloqueio de tela; c) Monitoramento do CS e; d) Controle parental do tempo de tela. Funções estas que corroboram as recomendações de estudos sobre CS, que aconselham a inclusão de intervenções comportamentais, monitoramento do tempo de tela e o envolvimento da família.

Palavras-chave: Estilo de Vida Sedentário. Tempo de tela. Apps Móveis.

Abstract: The objective of this study was to identify and group information from mobile applications aimed at reducing sedentary behavior (SB) existing on the Google Play and iTunes platforms. The survey took place in August 2020, using terms related to SB. Were found the 196 applications, most of which are available only in English and free. In general, applications can be divided into four categories: a) alerts/ reminders for breaking the SB; b) Application restriction and screen lock; c) SB monitoring; and d) Parental control of screen time. These functions corroborate the recommendations of studies on SB, which recommend the inclusion of behavioral interventions, monitoring screen time, and family involvement.

Keywords: Sedentary Lifestyle. Screen time. Mobile Apps.

Resumen: El objetivo de este estudio fue identificary agrupar información procedente de aplicaciones móviles orientadas a reducir las conductas sedentarias (CS) existentes en las plataformas Google Play y iTunes. La encuesta se llevó a cabo en agosto de 2020, utilizando términos relacionados con la informática. Se encontraron 196 aplicaciones, la mayoría de las cuales están disponibles solo en inglés y son gratuitas. En general, las aplicaciones se pueden dividir en cuatro categorías: a) alertas/recordatorios para romper la CS; b) Restricción de aplicaciones y bloqueo de pantalla; c) Monitoreo de SC y; d) Control parental del tiempo frente a la pantalla. Estas funciones corroboran las recomendaciones de estudios sobre CS, que recomiendan la inclusión de intervenciones 
conductuales, el seguimiento del tiempo frente a las pantallas y la participación familiar.

Palabras-clave: Estilo de vida sedentario. Tiempo de pantalla. Apps móviles.

Submetido em: 07-04-2021

Aceito em: 23-04-2021 


\section{Introdução}

Nas últimas décadas, verifica-se que a população mundial despende muito tempo em atividades de comportamento sedentário (CS) (EKELUND et al., 2016), sendo este definido como qualquer comportamento realizado em período de vigília em posição sentada, reclinada ou deitada, cujo gasto energético não ultrapasse 1,5 MET's - equivalentes metabólicos que representam a taxa de gasto energético da atividade (TREMBLAY et al., 2017). Dentre os fatores de risco para alto CS, o tempo gasto com dispositivos móveis se tornou uma preocupação mundial, visto que pessoas entre 18 e 80 anos de idade passam em média 4 horas por dia utilizando seus celulares e acumulando uma média de 8,7 horas por dia de tempo sentadas (FENNELL; BARKLEY; LEPP, 2019).

Evidências mostram que muito tempo despendido em CS, como assistir televisão por 5 horas ou mais por dia, está associado a um aumento entre $16,0 \%$ e $93,0 \%$ no risco de mortalidade por todas as causas, mesmo em pessoas que praticam níveis moderados e elevados de atividade física (EKELUND et al., 2016). Outros estudos também encontraram associações entre o elevado tempo despendido em CS e doenças cardiovasculares, câncer, diabetes tipo II e maior risco de mortalidade por todas as causas (BISWAS et al., 2015; KU et al., 2019; REZENDE et al., 2014).

Sendo o uso de mídias eletrônicas (tempo de tela) relacionado ao CS, intervenções de saúde vinculadas com a mídia desses aparelhos, como aplicativos e mensagens curtas, têm sido planejadas (COLL; AMORIM; HALLAL, 2010). Nesse sentido, o campo de Saúde Móvel (mobile health - mHealth), com o objetivo de oferecer e monitorar serviços de saúde por meio destes dispositivos (smartphones e aplicativos), vem sendo amplamente estudado por diversas razões: alta prevalência de uso de smartphone pela população, evolução constante das capacidades técnicas desses dispositivos, apego e tendência das pessoas carregarem o smartphone a todos os lugares, por permitirem o acesso às informações e permitir a 
Aplicativos móveis de saúde para redução do comportamento sedentário

Deisy Terumi Ueno • Angélica Bonolo • Jean Augusto Coelho Guimarães • Ana Elisa Messetti Christofoletti • Priscila Missaki Nakamura • Eduardo Kokubun

avaliação e feedback em qualquer hora ou lugar (ELAVSKY et al., 2019; KLASNJA; PRATT, 2012; LEWIS et al., 2017; LUPTON, 2014; MAS; SAMPOL; CONTI, 2016; ROCHA et al., 2016). Ainda, aplicativos destinados à saúde podem auxiliar na difusão dos conhecimentos e informações de práticas e hábitos saudáveis (CHAVES et al., 2018), tal qual ter um cunho educacional e inovador sobre o ensino-aprendizagem acerca da saúde (OLIVEIRA; ALENCAR, 2017).

Nessa perspectiva, é possível observar a crescente disponibilidade de aplicativos de saúde nos últimos anos (ROCHA et al., 2016). No ano de 2014, em um levantamento de aplicativos de saúde, os autores verificaram que havia 34.490 aplicativos disponíveis no iTunes e 17.756 no Google Play classificados no campo de saúde e fitness. No entanto, a maioria desses aplicativos concentrava-se na promoção de atividade física e alimentação saudável (MIDDELWEERD et al., 2014).

Em estudo de revisão sistemática de estudos de intervenção com aplicativos de saúde, Schoeppe et al., (2016), considerando que os comportamentos de saúde possuem determinantes e características específicas, apontaram que é importante conhecer as propriedades das intervenções de forma isolada, para permitir o desenvolvimento de novas intervenções e garantir a eficácia da intervenção para um determinado comportamento. Deste modo, o objetivo deste estudo foi identificar e agrupar informações e funções de aplicativos voltados para a redução do CS existentes nas plataformas do Google Play e iTunes. Ainda, o levantamento de tais informações permitirá a comparação dos achados com os estudos de intervenção com aplicativos para redução do CS existentes na literatura, assim como dar subsídios para futuras intervenções com a utilização de aplicativos voltados para a redução do CS.

\section{Métodos}

Este estudo é transversal de caráter exploratório e descritivo. No mês de agosto de 2020, realizou-se o levantamento dos apli- 
Aplicativos móveis de saúde para redução do comportamento sedentário

Deisy Terumi Ueno • Angélica Bonolo • Jean Augusto Coelho Guimarães • Ana Elisa Messetti Christofoletti • Priscila Missaki Nakamura • Eduardo Kokubun

cativos disponíveis nas bases de aplicativos Google Play e iTunes. Tais bases são referentes ao serviço de distribuição digital oficial de aplicativos para o sistema operacional Android e Apple, respectivamente, no qual o usuário procura pelo aplicativo ou funcionalidade de interesse e tem acesso a uma lista de aplicativos disponíveis para download.

A busca foi realizada por duas pesquisadoras, utilizando-se os termos relacionados ao CS, sedentary, sit, sitting time, screen time e downtime, sendo utilizado apenas um termo por vez. A escolha de tais termos foi baseada em termos relacionados ao CS e optou-se pela busca em inglês, visto que é considerado idioma universal e permite encontrar resultados em todos os idiomas.

\section{Critérios de inclusão e exclusão, Extração e Síntese dos dados}

Após a busca de aplicativos, a pesquisadora responsável pela busca na base do iTunes realizou a seleção dos aplicativos da base do Google Play e a pesquisadora que pesquisou a base do Google Play fez a seleção dos resultados da base iTunes, a opção pela troca para análise dos resultados ocorreu com o intuito de manter imparcialidade na seleção dos aplicativos. Durante as buscas, observou-se que as plataformas limitam a quantidade máxima de resultados apresentados, sendo 250 na base do Google Play e 100 no iTunes.

Em virtude da carência de estudos de relação sobre dose-resposta e CS (GUERRA; FARIAS JÚNIOR; FLORINDO, 2016), e como não há até o momento diretrizes específicas sobre intervenções com aplicativos de CS, a seleção das funções foi baseada na recomendação geral de que indivíduos devem realizar quebras (pausas) de pelo menos um minuto no CS (HEALY et al., 2008), de forma a minimizar os efeitos nocivos à saúde, assim como limitar o tempo de tela (FANNING et al., 2017; HEALY et al., 2008; LEVINE, 2004). A realização de atividades físicas de intensidade leve como formas de 
Aplicativos móveis de saúde para redução do comportamento sedentário

Deisy Terumi Ueno • Angélica Bonolo • Jean Augusto Coelho Guimarães • Ana Elisa Messetti Christofoletti • Priscila Missaki Nakamura • Eduardo Kokubun

promover as quebras são incentivadas por promoverem aumento no gasto energético diário e por mostrarem associação negativa mais forte com o CS do que as atividades com intensidades moderada e/ou vigorosa (HEALY et al., 2008).

Dessa forma, após a leitura da descrição dos aplicativos, foram selecionados os aplicativos que continham dentre suas funções a quebra/redução do CS, como bloqueio de tela, lembretes para levantar-se, entre outros.

Foram excluídos os aplicativos referentes à prática de atividade física, alimentação saudável e jogos, os quais apareceram nos resultados das buscas.

Ao final, as informações sobre: a) nome do aplicativo; b) desenvolvedor; c) categoria; d) preço; e) idioma e; f) as funções de redução de CS foram extraídas em planilha eletrônica Excel ${ }^{\circledR}$.

\section{Resultados}

Foram identificados 1.245 e 274 aplicativos nas bases Google Play e iTunes, respectivamente. Do total de 1.519 aplicativos encontrados, 1.323 foram excluídos após triagem pela descrição, os quais não reportavam função relacionada à redução de CS (1282; $84,4 \%)$ ou por estarem repetidos em cada base $(41 ; 2,7 \%)$, sendo selecionados assim 196 aplicativos (FIGURA 1). 
Aplicativos móveis de saúde para redução do comportamento sedentário

Deisy Terumi Ueno • Angélica Bonolo • Jean Augusto Coelho Guimarães • Ana Elisa Messetti Christofoletti • Priscila Missaki Nakamura • Eduardo Kokubun

Figura 1 - Fluxograma do processo de seleção dos aplicativos.

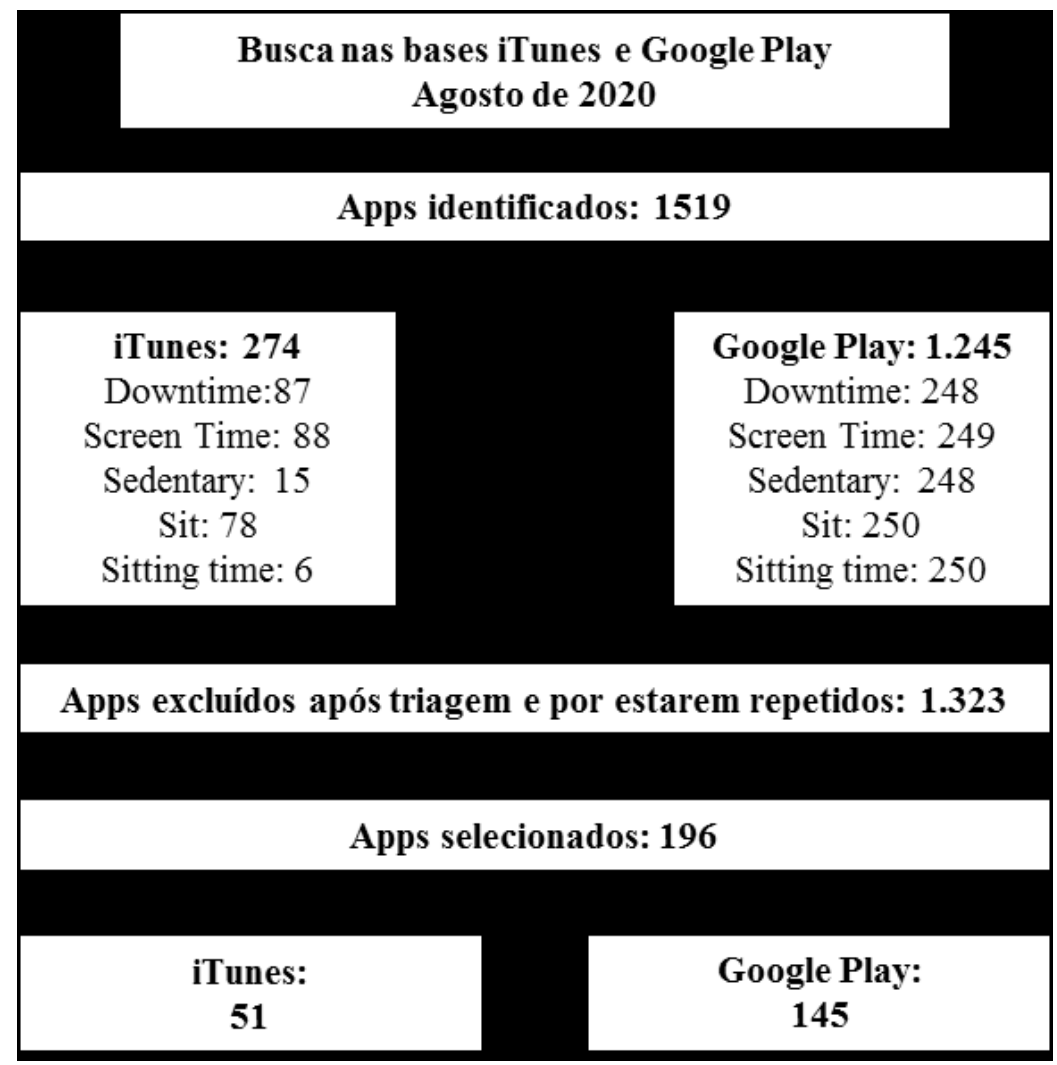

Fonte: Os autores (2021).

Do total de 196 aplicativos, 145 aplicativos estavam disponíveis na base do Google Play e 51 na base de aplicativos do iTunes. Em virtude de 16 aplicativos estarem disponíveis nas duas bases, os dados analisados e apresentados correspondem ao total de 180 aplicativos. Desses 180 aplicativos, $113(62,8 \%)$ continham apenas uma função, 46 (25,6\%) duas funções, 19 (10,6\%) três funções e 2 $(1,0 \%)$ todas as quatro funções. A descrição dos aplicativos (nome do aplicativo, desenvolvedor e funções) é apresentada no quadro 1 , no qual pode-se observar que $42(23,2 \%)$ dos aplicativos contêm a função de alerta/lembretes para movimentar-se; 68 (37,6\%) bloqueio de tela; 75 (41,4\%), monitoração do tempo de tela e; 85 $(47,0 \%)$ de controle parental, no qual os pais/responsáveis podem controlar o tempo de tela do dispositivo do(a) filho(a), funções estas para a quebra de CS. 
Aplicativos móveis de saúde para redução do comportamento sedentário

Deisy Terumi Ueno • Angélica Bonolo • Jean Augusto Coelho Guimarães • Ana Elisa Messetti Christo-

foletti • Priscila Missaki Nakamura • Eduardo Kokubun

Quadro 1. Características dos aplicativos das bases Google Play e iTunes.

\begin{tabular}{|c|c|c|c|c|c|}
\hline \multirow[b]{2}{*}{ Nome do APP } & \multirow[b]{2}{*}{ Desenvolvedor } & \multicolumn{4}{|c|}{ FUNÇÕES DE REDUÇÃO DO CS } \\
\hline & & $\begin{array}{l}\text { Alerta/ } \\
\text { Lembrete }\end{array}$ & $\begin{array}{l}\text { Bloqueio } \\
\text { de tela ou } \\
\text { app }\end{array}$ & $\begin{array}{l}\text { Monitoração } \\
\text { do tempo de } \\
\text { tela }\end{array}$ & $\begin{array}{l}\text { Controle } \\
\text { parental }\end{array}$ \\
\hline $\begin{array}{l}\text { AppGuardian (Versão Pais) - } \\
\text { Controle Parental A }\end{array}$ & AppGuardian & & & & $x$ \\
\hline $\begin{array}{l}\text { AScreen Timeout (Keep Screen } \\
\text { On) A }\end{array}$ & THSoftware & & $x$ & & \\
\hline Circle Parental Controls A & Circle Media, Inc & & & & $x$ \\
\hline $\begin{array}{l}\text { ActionDash: Bem-estar digital e } \\
\text { tempo de tela A }\end{array}$ & ActionDash & & $x$ & $x$ & \\
\hline Activator B & PAL Technologes Ltd & $x$ & & $x$ & \\
\hline $\begin{array}{l}\text { AntiSocial: dependência do } \\
\text { telefone A }\end{array}$ & Bugbean Pty Ltd & & $x$ & $x$ & \\
\hline App Qustodio para filhos B & Qustodio & & & & $x$ \\
\hline $\begin{array}{l}\text { App Usage - Controle o uso do } \\
\text { celular A }\end{array}$ & Sam Lu & $x$ & $x$ & $x$ & \\
\hline App Usage Tracker - Apps Time B & $\begin{array}{l}\text { Emberify } \\
\text { Innovations Private } \\
\text { Limited }\end{array}$ & & & $x$ & \\
\hline $\begin{array}{l}\text { App Use Time Tracker \& } \\
\text { Controller A }\end{array}$ & VDO apps & & $x$ & $x$ & \\
\hline $\begin{array}{l}\text { AppDetox - App Blocker for } \\
\text { Digital Detox A }\end{array}$ & AppDocs & $x$ & $x$ & & \\
\hline AppTime - App usage tracker A & Maximilian Nocker & & $x$ & $x$ & \\
\hline $\begin{array}{l}\text { Attention : Screen Time \& Stay } \\
\text { Focused A }\end{array}$ & $\begin{array}{l}\text { HD Wallpapers } \\
\text { Backgrounds Studio }\end{array}$ & & $x$ & $x$ & $x$ \\
\hline $\begin{array}{l}\text { Ava - Kid Mode Lock, Kid Security, } \\
\text { Screen Time App A }\end{array}$ & $\begin{array}{l}\text { iRIDIUM DUST } \\
\text { LIMITED }\end{array}$ & & & & $x$ \\
\hline Bakery - Study Focus Timer App B & Rob Norback & & $x$ & & \\
\hline Balance - Limit Screen Time A & Pyxsee Inc & $x$ & $x$ & $x$ & $x$ \\
\hline Bem-estar digital A & Google LLC & & $x$ & $x$ & \\
\hline Bitdefender Parental Control A & Bitdefender & & & & $x$ \\
\hline $\begin{array}{l}\text { Block Apps - Productivity \& Digital } \\
\text { Wellbeing A }\end{array}$ & Wverlaek & & $\mathrm{x}$ & $x$ & \\
\hline $\begin{array}{l}\text { Boomerang Parental Control - } \\
\text { Screen Time app A }\end{array}$ & $\begin{array}{l}\text { National Education } \\
\text { Technologies Inc. }\end{array}$ & & & & $x$ \\
\hline Break Reminder A & The Big Mom & $x$ & & & \\
\hline BreakFree - Track Screen Time! B & Saroj Rani Singla & $x$ & $\mathrm{x}$ & $x$ & \\
\hline $\begin{array}{l}\text { Cell Phone Addiction Timer - } \\
\text { Screen Time Unplugged B }\end{array}$ & JIANENG XUE & $x$ & $x$ & $x$ & \\
\hline $\begin{array}{l}\text { Chairless - Smart sitting time } \\
\text { tracker A }\end{array}$ & Runtime Error & $x$ & & $x$ & \\
\hline $\begin{array}{l}\text { Control Child Premium Parental } \\
\text { Control A }\end{array}$ & JET Yazılım & & & & $x$ \\
\hline $\begin{array}{l}\text { Controle Parental Kroha - Screen } \\
\text { Time app A, B }\end{array}$ & $\begin{array}{l}\text { Parental Control } \\
\text { Kroha }\end{array}$ & & $x$ & $x$ & $\mathrm{x}$ \\
\hline
\end{tabular}


Aplicativos móveis de saúde para redução do comportamento sedentário

Deisy Terumi Ueno • Angélica Bonolo • Jean Augusto Coelho Guimarães • Ana Elisa Messetti Christo-

foletti • Priscila Missaki Nakamura • Eduardo Kokubun

\begin{tabular}{|c|c|c|c|c|c|}
\hline \multirow[b]{2}{*}{ Nome do APP } & \multirow[b]{2}{*}{ Desenvolvedor } & \multicolumn{4}{|c|}{ FUNÇÕES DE REDUÇÃO DO CS } \\
\hline & & $\begin{array}{c}\text { Alerta/ } \\
\text { Lembrete }\end{array}$ & $\begin{array}{l}\text { Bloqueio de } \\
\text { tela ou app }\end{array}$ & $\begin{array}{c}\text { Monitoração } \\
\text { do tempo de } \\
\text { tela }\end{array}$ & $\begin{array}{l}\text { Controle } \\
\text { parental }\end{array}$ \\
\hline $\begin{array}{l}\text { Controle Parental de Qustodio } \\
\text { A, B }\end{array}$ & Qustodio & & & & $x$ \\
\hline $\begin{array}{l}\text { Controle Parental Screen Time } \\
\text { A, B }\end{array}$ & $\begin{array}{l}\text { Screen Time } \\
\text { Labs }\end{array}$ & & $x$ & & $x$ \\
\hline Deskfit ${ }^{A}$ & SelfShaper & $x$ & & & \\
\hline $\begin{array}{l}\text { Digitox: Bem-estar Digital - } \\
\text { tempo de tela }{ }^{A}\end{array}$ & Phosphorus & $x$ & & $x$ & \\
\hline DinnerTime Plus (Parenting) A & $\begin{array}{l}\text { NComputing } \\
\text { Global, Inc. }\end{array}$ & & $x$ & $x$ & $x$ \\
\hline Down Time ${ }^{A}$ & Dave Bennett & & $x$ & & \\
\hline Down Time ${ }^{A}$ & EvDevTech & & $x$ & & \\
\hline Downtime ${ }^{B}$ & $\begin{array}{l}\text { Adrian } \\
\text { Moorhouse }\end{array}$ & & & $x$ & \\
\hline Easy Parental Control A & $\begin{array}{l}\text { Khad Mobile } \\
\text { App }\end{array}$ & & & & $x$ \\
\hline ESET Parental Control A & ESET & & & & $x$ \\
\hline $\begin{array}{l}\text { Estatísticas do aplicativo: Uso de } \\
\text { rastreamento }{ }^{A}\end{array}$ & hhll tools & $x$ & $x$ & $x$ & \\
\hline Exsed $2^{\mathrm{B}}$ & $\begin{array}{l}\text { UKK } \\
\text { Terveyspalvelut }\end{array}$ & & & $x$ & \\
\hline $\begin{array}{l}\text { FamilyTime - Controlo Parental } \\
\text { A, B }\end{array}$ & Yumyapps & & & & $x$ \\
\hline FamilyTime Jr. ${ }^{A}$ & YumyApps & & & & $x$ \\
\hline Famisafe - Controle Parental A, B & $\begin{array}{l}\text { Wondershare } \\
\text { Technology } \\
\text { Group Co., LTD }\end{array}$ & & & & $x$ \\
\hline Fit While You Sit A, B & $\begin{array}{l}\text { Förlaget Tre Ben } \\
A B\end{array}$ & $x$ & & & \\
\hline FocusMe $^{A}$ & FocusMe Inc & $x$ & $x$ & & \\
\hline Forest: Mantenha o foco ${ }^{\mathrm{A}}$ & Seekrtech & & $x$ & & \\
\hline $\begin{array}{l}\text { Freedom | Bloqueie aplicativos } \\
\text { e sites distrativos A }\end{array}$ & $\begin{array}{l}\text { Eighty Percent } \\
\text { Solutions } \\
\text { Corporation }\end{array}$ & & $x$ & & \\
\hline $\begin{array}{l}\text { FunControl - the most fun } \\
\text { parental control app }{ }^{A}\end{array}$ & $\begin{array}{l}\text { BrainDrain } \\
\text { s.r.o. - parental } \\
\text { control apps }\end{array}$ & & & & $x$ \\
\hline Genius - Screen Lock For Kids ${ }^{B}$ & P-INK Ventures & & & & $x$ \\
\hline $\begin{array}{l}\text { Genius - Screen Time Parental } \\
\text { Control Remoto } 2020^{\mathrm{A}}\end{array}$ & $\begin{array}{l}\text { P-Ink Ventures } \\
\text { inc. }\end{array}$ & & & & $x$ \\
\hline $\begin{array}{l}\text { Google Family Link para crianças } \\
\text { e adolescentes }{ }^{\text {A }}\end{array}$ & Google LLC & & & & $x$ \\
\hline Google Family Link para pais ${ }^{A}$ & Google LLC & & & & $x$ \\
\hline $\begin{array}{l}\text { HelpMeFocus - Block Apps, Stay } \\
\text { Focused A }\end{array}$ & $\begin{array}{l}\text { dsimpletools } \\
\text { team }\end{array}$ & & $x$ & & \\
\hline
\end{tabular}


Aplicativos móveis de saúde para redução do comportamento sedentário

Deisy Terumi Ueno • Angélica Bonolo • Jean Augusto Coelho Guimarães • Ana Elisa Messetti Christofoletti • Priscila Missaki Nakamura • Eduardo Kokubun

Continuação quadro 1.

\begin{tabular}{|c|c|c|c|c|c|}
\hline \multirow[b]{2}{*}{ Nome do APP } & \multirow[b]{2}{*}{ Desenvolvedor } & \multicolumn{4}{|c|}{ FUNÇÕES DE REDUÇÃO DO CS } \\
\hline & & $\begin{array}{l}\text { Alerta/ } \\
\text { Lembrete }\end{array}$ & $\begin{array}{l}\text { Bloqueio de } \\
\text { tela ou app }\end{array}$ & $\begin{array}{c}\text { Monitoração } \\
\text { do tempo de } \\
\text { tela }\end{array}$ & $\begin{array}{l}\text { Controle } \\
\text { parental }\end{array}$ \\
\hline Hourly Fitness ${ }^{B}$ & Denis Beklarov & $x$ & & $\mathrm{x}$ & \\
\hline Instant - Lifelog Screen Time ${ }^{B}$ & $\begin{array}{l}\text { Emberify Innovations } \\
\text { Private Limited }\end{array}$ & & & $x$ & \\
\hline $\begin{array}{l}\text { Kaspersky SafeKids - Kids } \\
\text { mode }^{A}\end{array}$ & $\begin{array}{l}\text { Kaspersky Lab } \\
\text { Switzerland }\end{array}$ & & & & $x$ \\
\hline $\begin{array}{l}\text { Keep Me Focused - Beat } \\
\text { Phone Addiction }{ }^{A}\end{array}$ & JoeLabs & & $x$ & & \\
\hline Keep Me Out A & Vikesh Dass & & $x$ & & \\
\hline Kiddie Parental Control ${ }^{\mathrm{A}}$ & Frédéric Julian & & & & $x$ \\
\hline $\begin{array}{l}\text { Kids Home (Kids Mode, } \\
\text { Launcher, Parental Control) A }\end{array}$ & Arolle Solutions & & & & $x$ \\
\hline $\begin{array}{l}\text { Kids Launcher - Controle } \\
\text { Parental e Modo Infantil A }\end{array}$ & Gadestudios & & & & $x$ \\
\hline $\begin{array}{l}\text { Kids Mode - Child lock \& time } \\
\text { limit | Screen Time }{ }^{A}\end{array}$ & Innoxapps & & & & $x$ \\
\hline $\begin{array}{l}\text { Kids Place - Controle Parental } \\
\text { A }\end{array}$ & Kiddoware & & & & $x$ \\
\hline Kids Screen Time ${ }^{A}$ & Engineer Dad & & & & $\mathrm{x}$ \\
\hline $\begin{array}{l}\text { Kid's Shell lançador de } \\
\text { crianças controle parental A }\end{array}$ & Whisper Arts & & & & $x$ \\
\hline $\begin{array}{l}\text { Kids Zone - Parental Controls } \\
\text { \& Child Lock }{ }^{A}\end{array}$ & Out of the Park Apps & & & & $x$ \\
\hline Kidslox Controles dos Pais A, B & Kidslox Inc. & & & & $x$ \\
\hline KuuKla Ebeveyn Kontrol A & ARDIC INC. & & & & $x$ \\
\hline Leapteen - Parental Control B & Ding Zhang & & & & $x$ \\
\hline levantar lembrete ${ }^{\mathrm{B}}$ & Thang Nguyen & $x$ & & $\mathrm{x}$ & \\
\hline Lightdogs: Unplug \& Focus ${ }^{B}$ & Sapien Technology & & $x$ & & \\
\hline Limit Screen Time $^{A}$ & ScreenTime LLC & & $x$ & & \\
\hline Limitly Screen Time Control A & Limitly & & & & $x$ \\
\hline $\begin{array}{l}\text { Localizador Família Locategy } \\
\text { B }\end{array}$ & Locategy SL & & & & $x$ \\
\hline $\begin{array}{l}\text { Lock Me Out Freedom from } \\
\text { phone addiction A }\end{array}$ & TEQTIC & & $x$ & $x$ & \\
\hline $\begin{array}{l}\text { Luna - Kid Launcher \& } \\
\text { Parental controls }{ }^{\text {A }}\end{array}$ & AntTek Mobile & & & & $x$ \\
\hline Microsoft Family Safety A & $\begin{array}{l}\text { Microsoft } \\
\text { Corporation }\end{array}$ & & $x$ & $x$ & $x$ \\
\hline $\begin{array}{l}\text { MMGuardian Parental } \\
\text { Control App For Child Phone }{ }^{\text {A }}\end{array}$ & MMguardian.com & & & & $x$ \\
\hline $\begin{array}{l}\text { MMGuardian Parental } \\
\text { Control App For Parent } \\
\text { Phone }{ }^{\text {A }}\end{array}$ & MMguardian.com & & & & $x$ \\
\hline
\end{tabular}


Aplicativos móveis de saúde para redução do comportamento sedentário

Deisy Terumi Ueno • Angélica Bonolo • Jean Augusto Coelho Guimarães • Ana Elisa Messetti Christofoletti • Priscila Missaki Nakamura • Eduardo Kokubun

Continuação quadro 1.

\begin{tabular}{|c|c|c|c|c|c|}
\hline \multirow[b]{2}{*}{ Nome do APP } & \multirow[b]{2}{*}{ Desenvolvedor } & \multicolumn{4}{|c|}{ FUNÇÕES DE REDUÇÃO DO CS } \\
\hline & & $\begin{array}{l}\text { Alerta/ } \\
\text { Lembrete }\end{array}$ & $\begin{array}{l}\text { Bloqueio } \\
\text { de tela } \\
\text { ou app }\end{array}$ & $\begin{array}{l}\text { Monitoração do } \\
\text { tempo de tela }\end{array}$ & $\begin{array}{l}\text { Controle } \\
\text { parental }\end{array}$ \\
\hline $\begin{array}{l}\text { Mobicip Parental Control \& } \\
\text { Screen Time }{ }^{A, B}\end{array}$ & Mobicip & & & & $\mathrm{x}$ \\
\hline $\begin{array}{l}\text { Mobile Fence Parental Control } \\
\text { A }\end{array}$ & Mobile Fence & & & & $\mathrm{x}$ \\
\hline Mobile Screen Time Tracker ${ }^{A}$ & Vvstudio & & $x$ & $x$ & \\
\hline $\begin{array}{l}\text { Moment: Screen Time Control } \\
\mathrm{A}, \mathrm{B}\end{array}$ & Moment Health Inc. & & & $x$ & $x$ \\
\hline Monotony: Don't sit too long B & Assim Khan & $\mathrm{x}$ & & & \\
\hline Move Reminder ${ }^{A}$ & Endura Software & $x$ & & & \\
\hline $\begin{array}{l}\text { My Phone Time - App usage } \\
\text { tracking - Focus enabler }{ }^{A}\end{array}$ & Smarter Time & & $x$ & $x$ & \\
\hline $\begin{array}{l}\text { Neev - Kids mode \& Screen } \\
\text { time for Parents' phone }{ }^{A}\end{array}$ & Mithra Software & & & & $x$ \\
\hline $\begin{array}{l}\text { Net Nanny Parental Control } \\
\text { App }^{A, B}\end{array}$ & Zift Software LLC & & & & $x$ \\
\hline $\begin{array}{l}\text { Norton Family parental control } \\
\text { A }\end{array}$ & NortonMobile & & & & $x$ \\
\hline Off the Grid - Digital Detox ${ }^{A}$ & $\begin{array}{l}\text { TC International } \\
\text { Holdings, Inc. }\end{array}$ & & $\mathrm{x}$ & & \\
\hline OffScreen - Less Screen Time ${ }^{B}$ & Miidii Tech & & $x$ & $x$ & \\
\hline OFFTIME - Away Mobile ${ }^{\text {B }}$ & yuting dong & & $x$ & & \\
\hline $\begin{array}{l}\text { OFFTIME - Digital } \\
\text { disconnection }{ }^{A}\end{array}$ & mINdCUBEd & & $x$ & $x$ & \\
\hline $\begin{array}{l}\text { OurPact - Controle Parental } \\
\text { Screen Time A, B }\end{array}$ & Eturi Corp. & & & & $x$ \\
\hline OurPact Jr. ${ }^{\mathrm{B}}$ & Eturi Corp. & & & & $\mathrm{x}$ \\
\hline $\begin{array}{l}\text { ourValues Smarter Screen } \\
\text { Time \& Parental Control A }\end{array}$ & 3 Chiefs Pty Ltd & & & & $x$ \\
\hline $\begin{array}{l}\text { Owl Guard - Parental Control \& } \\
\text { Screen Time }{ }^{\text {A }}\end{array}$ & CZ Tech & & & & $x$ \\
\hline $\begin{array}{l}\text { Parent App - Limit Screen Time } \\
\text { A }\end{array}$ & Supotco Ltd & & & & $\mathrm{x}$ \\
\hline Parental Control - Kids Lock B & Jiangyong Liu & & & & $x$ \\
\hline $\begin{array}{l}\text { Parental Control: Screen Time } \\
\text { \& Location Tracker }{ }^{A}\end{array}$ & Bit Guardian GmbH & & & & $x$ \\
\hline $\begin{array}{l}\text { Parental Control App with Kid } \\
\text { Content by PikaPika }{ }^{A}\end{array}$ & $\begin{array}{l}\text { Floydwiz } \\
\text { Technologies Private } \\
\text { Limited }\end{array}$ & & & & $x$ \\
\hline $\begin{array}{l}\text { Parental Control CALMEAN for } \\
\text { children \& teens }{ }^{A}\end{array}$ & $\begin{array}{l}\text { Parental Control } \\
\& \text { Screen time - } \\
\text { CALMEAN }\end{array}$ & & & & $x$ \\
\hline $\begin{array}{l}\text { Parental Control Screen Time } \\
\text { Website Blocker }{ }^{A}\end{array}$ & Jane Moore Group & & & & $\mathrm{x}$ \\
\hline Parental Control SecureKids A & Grupo Deidev & & & & $\mathrm{x}$ \\
\hline
\end{tabular}


Aplicativos móveis de saúde para redução do comportamento sedentário

Deisy Terumi Ueno • Angélica Bonolo • Jean Augusto Coelho Guimarães • Ana Elisa Messetti Christofoletti • Priscila Missaki Nakamura • Eduardo Kokubun

Continuação quadro 1.

\begin{tabular}{|c|c|c|c|c|c|}
\hline \multirow[b]{2}{*}{ Nome do APP } & \multirow[b]{2}{*}{ Desenvolvedor } & \multicolumn{4}{|c|}{ FUNÇÕES DE REDUÇÃO DO CS } \\
\hline & & $\begin{array}{l}\text { Alerta/ } \\
\text { Lembrete }\end{array}$ & $\begin{array}{l}\text { Bloqueio } \\
\text { de tela } \\
\text { ou app }\end{array}$ & $\begin{array}{c}\text { Monitoração } \\
\text { do tempo de } \\
\text { tela }\end{array}$ & $\begin{array}{l}\text { Controle } \\
\text { parental }\end{array}$ \\
\hline $\begin{array}{l}\text { Parental Control: 'CALMEAN Control } \\
\text { Center'A }\end{array}$ & $\begin{array}{l}\text { Parental Control } \\
\text { \& Screen time - } \\
\text { CALMEAN }\end{array}$ & & & & $\mathrm{x}$ \\
\hline $\begin{array}{l}\text { Parental Controls App by Parental } \\
\text { Values }{ }^{A}\end{array}$ & Parental Values LLC. & & & & $x$ \\
\hline Parentsaround Parental Control ${ }^{\mathrm{A}}$ & ARS NOVA SYSTEMS & & & & $x$ \\
\hline $\begin{array}{l}\text { Phone App Usage Tracker - Monitor } \\
\text { Screen Time }{ }^{A}\end{array}$ & Aaru Soft Words & $\mathrm{x}$ & & $x$ & \\
\hline $\begin{array}{l}\text { Phone Usage Tracker - Monitor } \\
\text { your screen time }{ }^{A}\end{array}$ & Rake & & & $x$ & \\
\hline QualityTime - My Dieta Digital A & MobidaysApps & $\mathrm{x}$ & $\mathrm{x}$ & $\mathrm{x}$ & \\
\hline $\begin{array}{l}\text { Quantum: App Screen Time Stats - } \\
\text { Digital Wellbeing A }\end{array}$ & Peak Pocket Studios & & & $x$ & \\
\hline Rational Life-Screen Time Log ${ }^{B}$ & $\mathrm{Hao} \mathrm{Pu}$ & $x$ & & $x$ & \\
\hline RealizD - Screen Time Tracker ${ }^{\mathrm{B}}$ & RealizD Pty Ltd & & & $x$ & \\
\hline $\begin{array}{l}\text { Repeat Alarm - Recurring reminder } \\
\text { A }\end{array}$ & Dan's Inc & $x$ & & & \\
\hline RescueTime ${ }^{\text {B }}$ & Rescue Time & $\mathrm{x}$ & & $x$ & \\
\hline Rest timer ${ }^{A}$ & Lucidiem & $x$ & & $\mathrm{x}$ & \\
\hline Rise \& Recharge ${ }^{\mathrm{B}}$ & $\begin{array}{l}\text { Baker IDI Heart \& } \\
\text { Diabetes Institute }\end{array}$ & $\mathrm{x}$ & & $x$ & \\
\hline $\begin{array}{l}\text { Router Limits - Screen Time } \\
\text { Management }{ }^{A}\end{array}$ & Router Limits & & $x$ & $x$ & \\
\hline $\begin{array}{l}\text { Safe Family - Aplicativo de controle } \\
\text { dos pais }^{A}\end{array}$ & McAfee LLC & & & & $x$ \\
\hline Safe Family ${ }^{\text {B }}$ & McAfee, LLC. & & & & $x$ \\
\hline $\begin{array}{l}\text { Safe Lagoon - Parental Control with } \\
\text { Advanced Al }{ }^{\mathrm{A}}\end{array}$ & Safe Lagoon Software & & & & $x$ \\
\hline SaferKid Text Monitoring: Text ${ }^{\mathrm{B}}$ & SaferKid, Inc. & & $x$ & & $\mathrm{x}$ \\
\hline $\begin{array}{l}\text { Screen Stopwatch - A Digital } \\
\text { Wellbeing Experiment }{ }^{A}\end{array}$ & Google Creative Lab & & & $x$ & \\
\hline Screen Time ${ }^{A}$ & Simple App Ltd. & & $x$ & $x$ & \\
\hline Screen Time - Digital Wellbeing A & Goodboy & $x$ & & $x$ & \\
\hline $\begin{array}{l}\text { Screen Time - Limit Time Usage in } \\
\text { Apps }{ }^{A}\end{array}$ & SmartApp Ltd & & $x$ & $x$ & \\
\hline $\begin{array}{l}\text { Screen Time - Media Time Manager } \\
\text { B }\end{array}$ & Rod Liberal & & & & $x$ \\
\hline Screen Time - Parental Control ${ }^{\mathrm{B}}$ & Cantina Software & $\mathrm{x}$ & & & \\
\hline $\begin{array}{l}\text { Screen Time - Restringir-se e } \\
\text { controle dos pais }{ }^{A}\end{array}$ & AppBuck3t & $x$ & $x$ & $\mathrm{x}$ & \\
\hline Screen Time ${ }^{A}$ & Yoav Sabag & & & $\mathrm{x}$ & \\
\hline
\end{tabular}


Aplicativos móveis de saúde para redução do comportamento sedentário

Deisy Terumi Ueno • Angélica Bonolo • Jean Augusto Coelho Guimarães • Ana Elisa Messetti Christofoletti • Priscila Missaki Nakamura • Eduardo Kokubun

Continuação quadro 1.

\begin{tabular}{|c|c|c|c|c|c|}
\hline \multirow[b]{2}{*}{ Nome do APP } & \multirow[b]{2}{*}{ Desenvolvedor } & \multicolumn{4}{|c|}{ FUNÇÕES DE REDUÇÃO DO CS } \\
\hline & & $\begin{array}{l}\text { Alerta/ } \\
\text { Lembrete }\end{array}$ & $\begin{array}{l}\text { Bloqueio } \\
\text { de tela ou } \\
\text { app }\end{array}$ & $\begin{array}{c}\text { Monitoração } \\
\text { do tempo de } \\
\text { tela }\end{array}$ & $\begin{array}{l}\text { Controle } \\
\text { parental }\end{array}$ \\
\hline Screen time ${ }^{B}$ & BabyBinks & $x$ & & $x$ & \\
\hline Screen time Challenge ${ }^{\mathrm{B}}$ & Kidslock Trading Ltd & $x$ & $x$ & $x$ & \\
\hline Screen Time Control ${ }^{\mathrm{A}}$ & Shexa Technolabs & $x$ & $x$ & $x$ & \\
\hline Screen Time Control ${ }^{\mathrm{A}}$ & Bazzinga Apps & & $x$ & & \\
\hline $\begin{array}{l}\text { Screen Time Control: } \\
\text { ZenScreen }{ }^{A, B}\end{array}$ & Zen Labs, Inc & & $x$ & $x$ & $x$ \\
\hline $\begin{array}{l}\text { Screen Time- Controle } \\
\text { Parental e Restrição Pessoal } \\
\text { A, B }\end{array}$ & $\begin{array}{l}\text { IRIDIUM DUST } \\
\text { LIMITED }\end{array}$ & & $x$ & $x$ & $x$ \\
\hline Screen Time for Kids ${ }^{A}$ & ScreenTime LLC & $\mathrm{x}$ & $\mathrm{x}$ & $x$ & $x$ \\
\hline $\begin{array}{l}\text { Screen Time iOS } 12 \text { - Phone } 11 \\
\text { Screen Time }^{A}\end{array}$ & Quynh BupBe & & & & $x$ \\
\hline Screen Time Limit KidCrono ${ }^{B}$ & RecreaVision & & & & $x$ \\
\hline Screen time logger ${ }^{A}$ & Kristoffer Geyer & & & $x$ & \\
\hline $\begin{array}{l}\text { Screen Time Parental Control } \\
\text { \& Parents Child Lock }{ }^{\mathrm{B}}\end{array}$ & Catrnja Dev & & & & $x$ \\
\hline Screen Time Self Control B & xueping cai & & $x$ & $x$ & \\
\hline Screen Time Squish ${ }^{A}$ & Rimbe INC & & & & $x$ \\
\hline $\begin{array}{l}\text { Screen Time Tracker - } \\
\text { Wellbeing A }\end{array}$ & KING Studio & & $x$ & $x$ & \\
\hline Screen Timeout A & Ihoer0 & & $x$ & & \\
\hline $\begin{array}{l}\text { Screen Timer - Stop Wasting } \\
\text { Time }^{A}\end{array}$ & Rodolfo Solano & $x$ & $\mathrm{x}$ & & \\
\hline $\begin{array}{l}\text { ScreenGuide Controle } \\
\text { Parental } A, B\end{array}$ & Mosyle Corporation & & & & $\mathrm{x}$ \\
\hline $\begin{array}{l}\text { SecureTeen Parental Control } \\
A, B\end{array}$ & Infoweise Pty. Ltd & & & & $x$ \\
\hline Sentry Parental Control A & LidrTech Ltd. & & & & $x$ \\
\hline Sitting Timer ${ }^{A}$ & Felno & $x$ & $x$ & $x$ & \\
\hline $\begin{array}{l}\text { Skreentime - Detox from } \\
\text { internet and limit apps }{ }^{A}\end{array}$ & STL software & $x$ & $x$ & $x$ & \\
\hline $\begin{array}{l}\text { Smarter Time - Time } \\
\text { Management - Productivity }{ }^{A}\end{array}$ & Smarter Time & & $x$ & $x$ & \\
\hline $\begin{array}{l}\text { SocialX - Appp para rastrear o } \\
\text { tempo de tela Android }{ }^{A}\end{array}$ & FunSwitch & & $x$ & $x$ & \\
\hline $\begin{array}{l}\text { Sour Note - Screen Time } \\
\text { Reminder }^{A}\end{array}$ & SourDevelopment & $x$ & & & \\
\hline $\begin{array}{l}\text { Sowi - Screen Time Balance } \\
\text { for Kids }{ }^{\text {A }}\end{array}$ & Sowi & & & & $x$ \\
\hline $\begin{array}{l}\text { SPACE - Break phone } \\
\text { addiction }^{B}\end{array}$ & Mrigaen Kapadia & & & $x$ & \\
\hline
\end{tabular}


Aplicativos móveis de saúde para redução do comportamento sedentário

Deisy Terumi Ueno • Angélica Bonolo • Jean Augusto Coelho Guimarães • Ana Elisa Messetti Christofoletti • Priscila Missaki Nakamura • Eduardo Kokubun

Continuação quadro 1.

\begin{tabular}{|c|c|c|c|c|c|}
\hline \multirow[b]{2}{*}{ Nome do APP } & \multirow[b]{2}{*}{ Desenvolvedor } & \multicolumn{4}{|c|}{ FUNÇÕES DE REDUÇÃO DO CS } \\
\hline & & $\begin{array}{l}\text { Alerta/ } \\
\text { Lembrete }\end{array}$ & $\begin{array}{l}\text { Bloqueio } \\
\text { de tela ou } \\
\text { app }\end{array}$ & $\begin{array}{c}\text { Monitoração } \\
\text { do tempo de } \\
\text { tela }\end{array}$ & $\begin{array}{l}\text { Controle } \\
\text { parental }\end{array}$ \\
\hline $\begin{array}{l}\text { SPACE - Rompa com o vício do } \\
\text { telefone }{ }^{A}\end{array}$ & Mobifolio & $x$ & $x$ & $x$ & \\
\hline ST Kids App ${ }^{A}$ & Screen Time Labs & & & & $x$ \\
\hline Stand up $\mathrm{PH}^{\mathrm{A}}$ & Kerchlynn Tan & $x$ & & & \\
\hline Stand up tracker lembrete ${ }^{A}$ & $\begin{array}{l}\text { VICTO IDEAS Health } \\
\text { \& Fitness Lab }\end{array}$ & $x$ & & $x$ & \\
\hline $\begin{array}{l}\text { Stay Away: Phone Addiction } \\
\text { Controller Antisocial }{ }^{\text {A }}\end{array}$ & Stay Away & & $x$ & $x$ & \\
\hline $\begin{array}{l}\text { Stay Focused - Bloco de } \\
\text { aplicativos bloco de sites }{ }^{A}\end{array}$ & Innoxapps & $x$ & $x$ & $x$ & \\
\hline $\begin{array}{l}\text { StayAway (PhoneLock): } \\
\text { KeepMeOut from Phone } \\
{\text { addiction }{ }^{A}}\end{array}$ & SleepTech & & $x$ & & \\
\hline $\begin{array}{l}\text { StayFree - Bem-estar digital e } \\
\text { tempo de tela A }\end{array}$ & StayFree Apps & $x$ & & $x$ & \\
\hline Stopsitting A & Eldar Mamedov & $x$ & & $x$ & \\
\hline $\begin{array}{l}\text { Teen Time - Parental Control, } \\
\text { Screen Time \& GPS }{ }^{\text {A }}\end{array}$ & $\begin{array}{l}\text { Parental Control } \\
\text { App by Teen Time }\end{array}$ & & $x$ & $x$ & $x$ \\
\hline Teen Time for Kids ${ }^{A}$ & $\begin{array}{l}\text { Parental Control } \\
\text { App by Teen Time }\end{array}$ & & & & $x$ \\
\hline Tempo de uso de telefone ${ }^{A}$ & JK.Fantasy & & & $x$ & \\
\hline TigerMom - Parental Control A & $\begin{array}{l}\text { Trustlook Security } \\
\text { Lab }\end{array}$ & & & & $x$ \\
\hline $\begin{array}{l}\text { Time Creator - track phone } \\
\text { usage }{ }^{\text {A }}\end{array}$ & Malte Wabnitz & $x$ & & $x$ & \\
\hline Timed screen-lock ${ }^{A}$ & Afakan & & $x$ & & \\
\hline $\begin{array}{l}\text { TimeLimit.io - free usage } \\
\text { duration limitation }{ }^{A}\end{array}$ & JoLo Software & & $x$ & & \\
\hline $\begin{array}{l}\text { Timeout - Control Your } \\
\text { Smartphone Addiction A }\end{array}$ & 3Q Consulting & & $x$ & $x$ & \\
\hline Tittle Parental Control A, B & Tittle for Parents & & & & $x$ \\
\hline $\begin{array}{l}\text { Toddler Lock Timer - For Kids } \\
\text { under } 6 \text { A }\end{array}$ & $\begin{array}{l}\text { BC Optima } \\
\text { Technologies }\end{array}$ & & & & $x$ \\
\hline ToilSafe YT Kids \& Screen time ${ }^{\text {B }}$ & Kids1st Inc. & & & & $x$ \\
\hline $\begin{array}{l}\text { Usage Time - App Usage } \\
\text { Manager }^{A}\end{array}$ & Vnova Mobile & & $x$ & $x$ & \\
\hline Usage Time. Smartphonoholic A & Jackypot & & $x$ & $x$ & \\
\hline $\begin{array}{l}\text { UsageSafe: App Blocker and } \\
\text { Phone Usage Tracker }{ }^{A}\end{array}$ & CloudDroid & & $x$ & $x$ & \\
\hline $\begin{array}{l}\text { Uso do telefone: monitor de } \\
\text { tempo de tela }{ }^{A}\end{array}$ & $\begin{array}{l}\text { Bubble Apps } \\
\text { Productivity Tools }\end{array}$ & & $x$ & $x$ & \\
\hline $\begin{array}{l}\text { YourHour - Phone Addiction } \\
\text { Tracker \& Controller }{ }^{A}\end{array}$ & Mindefy Labs & & & $x$ & \\
\hline Yubi: Kids Control for Family ${ }^{\mathrm{B}}$ & EonScope Inc & & & & $x$ \\
\hline
\end{tabular}

Nota: A Google Play app; B iTunes app; CS Comportamento Sedentário. Fonte: Os autores (2021). 
Aplicativos móveis de saúde para redução do comportamento sedentário

Deisy Terumi Ueno • Angélica Bonolo • Jean Augusto Coelho Guimarães • Ana Elisa Messetti Christofoletti • Priscila Missaki Nakamura • Eduardo Kokubun

Em geral, a maior fração dos aplicativos $(53 ; 29,3 \%)$ se encontra na categoria "Produtividade", seguido de "Criar os filhos" (33; 18,2\%), "Ferramentas" (31; 17,1\%), "Estilo de vida" (21; 11,6\%), "Saúde e Fitness" (21; 11,6\%), "Educação" (7; 3,9\%), "Utilidades" (6; 3,3\%), "Personalização" (4; 2,2\%), "Entretenimento" (3; 1,7\%), e "Jogos" (1; 0,6\%) (Figura 2).

Figura 2. Categorias dos aplicativos de acordo com as bases de aplicativos.

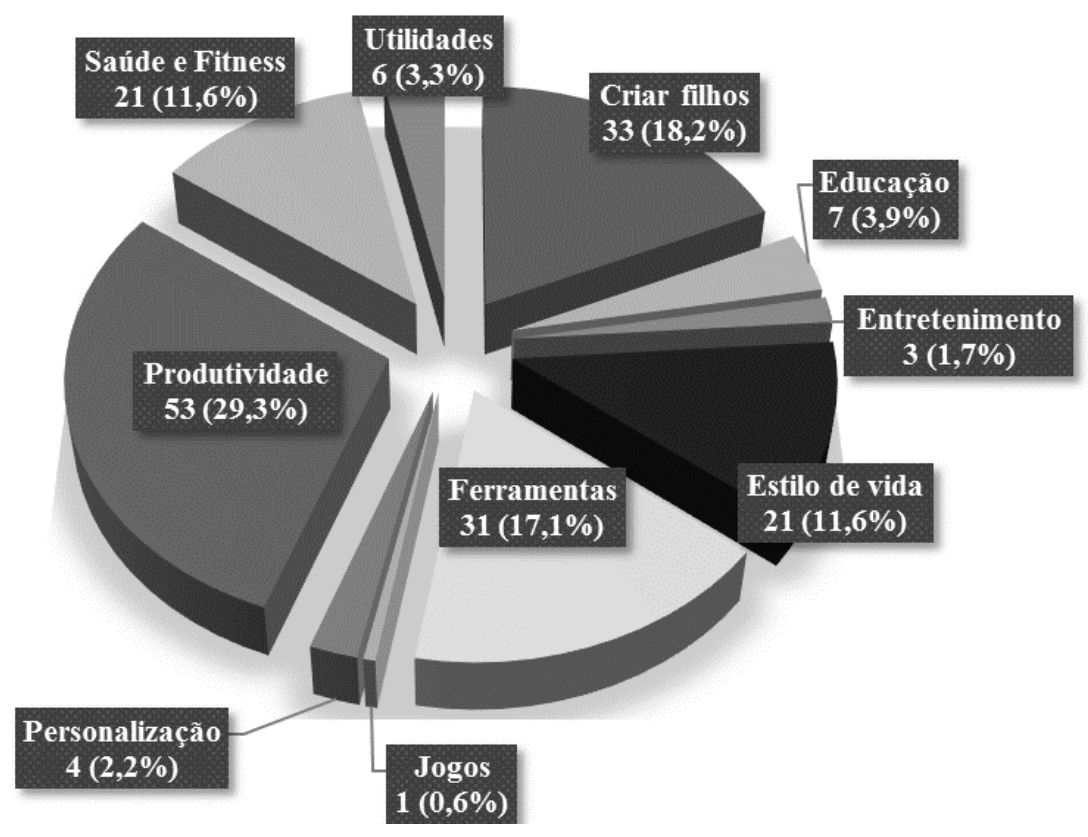

Fonte: Os autores (2021).

Com relação ao idioma, verificamos que 162 (89,5\%) dos aplicativos estão disponíveis em inglês, 29 (16,0\%) em português e 29 $(16,0 \%)$ em dois ou mais idiomas, podendo conter o idioma inglês e/ou português também (Figura 3). A grande maioria dos aplicativos $(171 ; 94,5 \%)$ está disponível de forma gratuita, sendo mais prevalente no Google Play. Os aplicativos pagos (29; 16\%) possuem valor mínimo de $\mathrm{R} \$ 3,90$ e máximo de $\mathrm{R} \$ 849$, 90 e são mais prevalentes na base do iTunes (Figura 4). 
Aplicativos móveis de saúde para redução do comportamento sedentário

Deisy Terumi Ueno • Angélica Bonolo • Jean Augusto Coelho Guimarães • Ana Elisa Messetti Christofoletti • Priscila Missaki Nakamura • Eduardo Kokubun

Figura 3. Idiomas dos aplicativos

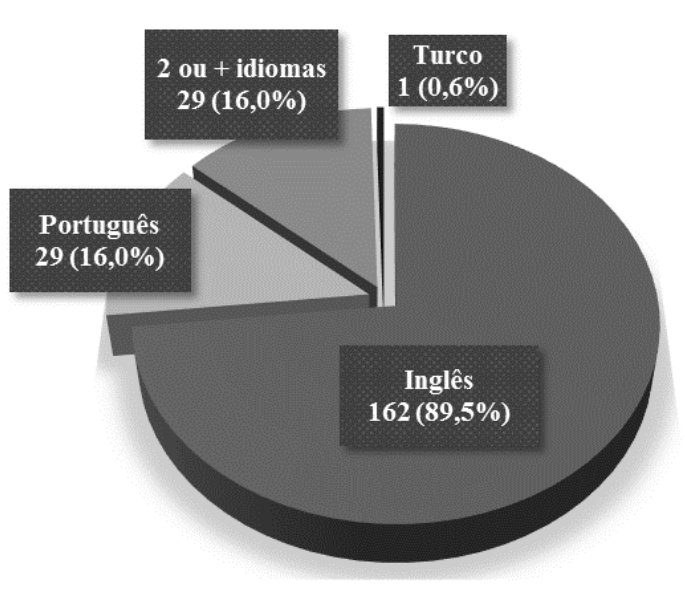

Fonte: Os autores (2021).
Figura 4. Aplicativos pagos e gratuitos

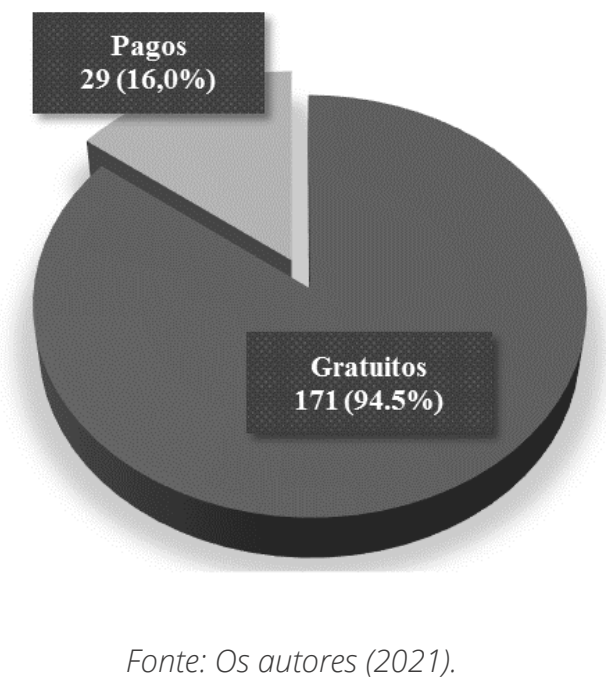

\section{Discussão}

Este estudo teve como objetivo identificar e agrupar informações e funções de aplicativos voltados para a redução do CS existentes nas plataformas do Google Play e iTunes. Assim, no ano de 2020, encontramos 145 no Google Play e 51 aplicativos disponíveis no iTunes. Contudo, é importante ressaltar que as bases de aplicativos tendem a limitar a quantidade máxima de resultados apresentados (250 no Google Play e 100 no iTunes) independente do pesquisador. Dessa forma, pode ser que muitos aplicativos disponíveis relacionados não tenham sido encontrados em nossas buscas.

Em geral, os aplicativos podem ser divididos em quatro categorias de acordo com as funções para redução do CS: a) Restrição de aplicativos e bloqueio de tela - desenvolvidos para melhorar o rendimento no trabalho e diminuir o tempo gasto em tela; b) Alertas/lembretes para quebra do CS - emite alerta de tempo de uso após determinado tempo; c) monitoramento do CS - para ser utilizado junto com algum monitor ou por inserção manual das informações referentes ao tempo despendido em CS, o qual gera 
Aplicativos móveis de saúde para redução do comportamento sedentário

Deisy Terumi Ueno • Angélica Bonolo • Jean Augusto Coelho Guimarães • Ana Elisa Messetti Christofoletti • Priscila Missaki Nakamura • Eduardo Kokubun

um gráfico diário ou semanal que permite o acompanhamento e comparação do comportamento ao longo dos dias e; d) Controle parental - permitem os pais e/ou responsáveis a controlar o tempo de tela e aplicativos instalados no dispositivo do(a) filho(a).

A função de restrição de aplicativos permite que o usuário bloqueie temporariamente o acesso a aplicativos ou até mesmo o aparelho, de forma que o usuário não consiga acessar qualquer aplicativo ou configuração durante o tempo pré-configurado. Considerando que a intenção (propósito) está relacionada à maioria dos tipos de atividades sedentárias de lazer, Rhodes e Dean (2009) acrescentam que estratégias que incluam a tomada de decisão podem ser viáveis nas diminuição do CS. Deste modo, essa função permite que o indivíduo configure a restrição de aplicativos e/ou do aparelho, para que possa realizar quebras (pausas), e assim minimizar os efeitos nocivos advindos do alto tempo despendido em CS, em especial com o uso do smartphone.

Com relação à função em especial de controle parental, verifica-se que esta é voltada principalmente para o controle do tempo de tela de crianças e adolescentes, visto que permite aos pais e/ ou responsáveis limitar o tempo de tela de seus filhos, além de assegurar se os conteúdos acessados são adequados à faixa etária e possíveis conversas com pessoas desconhecidas. Em estudo de revisão sobre intervenções para diminuição de CS em crianças e adolescentes, os achados mostraram as intervenções para crianças em idade pré-escolar que incluíram o envolvimento familiar, intervenções comportamentais e de controle do tempo de tela que foram mais favoráveis e podem estar relacionados a um maior sucesso da intervenção, quando comparados a outros estudos de intervenção (BIDDLE; PETROLINI; PEARSON, 2014). Dessa forma, infere-se que tal funcionalidade pode auxiliar no controle do tempo de tela de crianças e adolescentes em especial, desde que haja a preocupação primária dos pais e/ou responsáveis.

Visto que o CS é um problema de saúde recente, as intervenções para redução do CS estão em crescente evolução e a recomendação de futuras intervenções dependem da compreensão 
Aplicativos móveis de saúde para redução do comportamento sedentário

Deisy Terumi Ueno • Angélica Bonolo • Jean Augusto Coelho Guimarães • Ana Elisa Messetti Christofoletti • Priscila Missaki Nakamura • Eduardo Kokubun

do que funciona na mudança de CS (GARDNER et al., 2016). Desse modo, devido à falta de diretrizes específicas para o CS e de carência de estudos com aplicativos específicos para a redução de CS, os achados do presente estudo foram comparados com as recomendações de estudos de intervenção de CS existentes. Assim, podemos considerar que as funções presentes nos aplicativos vão ao encontro da recomendação de que as pessoas devem limitar o tempo de tela e diminuir o tempo gasto em CS (FANNING et al., 2017; HEALY et al., 2008; LEVINE, 2004), e que devem incluir o envolvimento da família, com intervenções comportamentais e monitoramento (limite) do tempo de tela (GARDNER et al., 2016; MANINI et al., 2015).

Estudos com aplicativos voltados para a redução do CS têm demonstrado resultados satisfatórios com baixo custo (LITMAN et al., 2015; LUBANS et al., 2014). No entanto, em estudo de revisão, Schoeepe et al., (2016) apontam que aplicativos multicomponentes, voltados à prevenção do CS e promoção de atividade física e alimentação saudável, parecem apresentar melhores resultados do que os aplicativos independentes.

Apesar de estudos com aplicativos de saúde em geral mostrarem boas evidências, podendo auxiliar na propagação dos conhecimentos e informações de práticas e hábitos saudáveis (CHAVES et al., 2018), os aplicativos, suas funções e efeitos comportamentais ainda apresentam resultados que carecem de maiores estudos que legitimem seu uso na área da saúde e que investiguem as limitações das medidas e metodologias desses aplicativos, de modo a entender por que há um declínio na utilização dos aplicativos ao longo do tempo, assim como também o potencial dessas intervenções a longo prazo (BUCKINGHAM et al., 2019; FANNING et al., 2017; LEWIS et al., 2017; MCINTOSH et al., 2017). Além disso, é importante investigar também o padrão de comportamento das diferentes faixas etárias com o uso de dispositivos móveis e com aplicativos de saúde, de modo a entender os padrões de comportamento, as preferências e eficácia de cada funcionalidade em cada público etário. 
Aplicativos móveis de saúde para redução do comportamento sedentário

Deisy Terumi Ueno • Angélica Bonolo • Jean Augusto Coelho Guimarães • Ana Elisa Messetti Christofoletti • Priscila Missaki Nakamura • Eduardo Kokubun

Dentre as limitações do presente estudo, podemos apontar a limitação das plataformas Google Play e iTunes, que restringem os resultados em 250 e 100, respectivamente, independente do pesquisador. Dessa forma, pode ser que nosso estudo não tenha contemplado todos os aplicativos relacionados que estão disponíveis nessas bases. Por sua vez, dentre os pontos fortes, podemos destacar o pioneirismo deste estudo em verificar aplicativos voltados à redução de CS disponíveis nas plataformas, os quais podem auxiliar seus usuários na diminuição do CS. Além disso, mostra a preocupação em desenvolver intervenções voltadas para a redução do CS não só de pesquisadores da área da saúde, mas também de outros profissionais, como as empresas de desenvolvimento de aplicativos.

\section{Conclusão}

Em nossa busca nas plataformas Google Play e iTunes, foi possível identificar 196 aplicativos voltados para a redução de CS. Tais aplicativos têm como funções principais a restrição no tempo de uso de aplicativos ou do dispositivo móvel, temporizador e alertas/ lembretes para quebra do CS, monitoramento do tempo gasto em CS e controle parental que permitem os pais controlarem o tempo de uso e os aplicativos utilizados pelo(a) filho(a).

De forma geral, os aplicativos são, em grande parte, gratuitos, contêm funções que parecem ser de fácil adoção e que permitem o incentivo à realização de quebras e/ou por meio da restrição no tempo de tela, os quais têm sido recomendados grandemente por pesquisadores de CS, de forma a promover o maior gasto energético total. No entanto, estudos sobre a real eficácia desses aplicativos a curto e a longo prazo ainda são necessários, visto que a utilização desses aplicativos pode auxiliar as pessoas a monitorarem e controlarem o tempo gasto em tela, problema este de caráter mundial e que vem sendo associado a diversos problemas de saúde da população. 
Aplicativos móveis de saúde para redução do comportamento sedentário

Deisy Terumi Ueno • Angélica Bonolo • Jean Augusto Coelho Guimarães • Ana Elisa Messetti Christofoletti • Priscila Missaki Nakamura • Eduardo Kokubun

\section{Referências}

BIDDLE, Stuart J. H.; PETROLINI, Irene; PEARSON, Natalie.

Interventions designed to reduce sedentary behaviours in young people: A review of reviews. British Journal of Sports Medicine, v. 48, n. 3, p. 182-186, 2014. DOI: 10.1136/bjsports-2013-093078. BISWAS, Aviroop; OH, Paul I.; FAULKNER, Guy E.; BAJAJ, Ravi R.; SILVER, Michael A.; MITCHELL, Marc S.; ALTER, David A. Sedentary time and its association with risk for disease incidence, mortality, and hospitalization in adults a systematic review and meta-analysis. Annals of Internal Medicine, v. 162, n. 2, p. 123-132, 2015. DOI: 10.7326/M14-1651.

BUCKINGHAM, Sarah Ann; WILLIAMS, Andrew James; MORRISSEY, Karyn; PRICE, Lisa; HARRISON, John. Mobile health interventions to promote physical activity and reduce sedentary behaviour in the workplace: A systematic review. Digital Health, v. 5, p. 1-50, 2019. DOI: 10.1177/2055207619839883.

CHAVES, Arlane Silva Carvalho; OLIVEIRA, Garithuzy Macedo; JESUS, Layane Mota de Souza De; MARTINS, Jose Lauro; SILVA, Valdirene Cássia Da. Uso de aplicativos para dispositivos móveis no processo de educação em saúde. Humanidades e Inovação, Palmas, v. 5, n. 6, p. 34-42, 2018. Disponível em: https://revista. unitins.br/index.php/humanidadeseinovacao/article/view/744.

COLL, C. V.; AMORIM, T. C.; HALLAL, P. C. Percepção De Adolescentes E Adultos Referente À Influência Da Mídia Sobre O Estilo De Vida. Revista Brasileira de Atividade Física e Saúde, v. 15, n. 2, p. 105-110, 2010. DOI: 10.12820/rbafs.v.15n2p105-110. EKELUND, UIf; STEENE-JOHANNESSEN, Jostein; BROWN, Wendy J.; FAGERLAND, Morten Wang; OWEN, Neville; POWELL, Kenneth E.; BAUMAN, Adrian; LEE, I. Min. Does physical activity attenuate, or even eliminate, the detrimental association of sitting time with mortality? A harmonised meta-analysis of data from more than 1 million men and women. The Lancet, v. 388, n. 10051, p. 1302- 
Aplicativos móveis de saúde para redução do comportamento sedentário

Deisy Terumi Ueno • Angélica Bonolo • Jean Augusto Coelho Guimarães • Ana Elisa Messetti Christofoletti • Priscila Missaki Nakamura • Eduardo Kokubun

1310, 2016. DOI: 10.1016/S0140-6736(16)30370-1. Disponível em: http://dx.doi.org/10.1016/S0140-6736(16)30370-1.

ELAVSKY, Steriani; KNAPOVA, Lenka; KLOCEK, Adam; SMAHEL, David. Mobile Health Interventions for Physical Activity, Sedentary Behavior, and Sleep in Adults Aged 50+: A Systematic Literature Review. Journal of Aging and Physical Activity, v. 27, n. 4, p. 565-593, 2019.

FANNING, Jason; ROBERTS, Sarah; HILLMAN, Charles H.; MULLEN, Sean P.; RITTERBAND, Lee; MCAULEY, Edward. A smartphone "app"-delivered randomized factorial trial targeting physical activity in adults. Journal of Behavioral Medicine, v. 40, n. 5, p. 712-729, 2017. DOI: 10.1007/s10865-017-9838-y.

FENNELL, Curtis; BARKLEY, Jacob E.; LEPP, Andrew. The relationship between cell phone use, physical activity, and sedentary behavior in adults aged 18-80. Computers in Human Behavior, v. 90 , n. July 2018 , p. 53-59, 2019. DOI: 10.1016/j.chb.2018.08.044. Disponível em: https://doi.org/10.1016/j.chb.2018.08.044.

GARDNER, Benjamin; SMITH, Lee; LORENCATTO, Fabiana; HAMER, Mark; BIDDLE, Stuart J. H. How to reduce sitting time? A review of behaviour change strategies used in sedentary behaviour reduction interventions among adults. Health Psychology Review, v. 10, n. 1, p. 89-112, 2016. DOI: 10.1080/17437199.2015.1082146.

GUERRA, Paulo Henrique; FARIAS JÚNIOR, José Cazuza; FLORINDO, Alex Antonio. Sedentary behavior in Brazilian children and adolescents: a systematic review. Revista de saude publica, São Paulo, v. 50, p. 9, 2016. DOI: 10.1590/S15188787.2016050006307.

HEALY, Genevieve N.; DUNSTAN, David W.; SALMON, Jo; CERIN, Ester; SHAW, Jonathan; ZIMMET, Paul; OWEN, Neville. Breaks in Sedentary time - Beneficial associations with metabolic risk. Diabetes Care, v. 31, n. 4, p. 661-666, 2008. DOI: 10.2337/dc072046.Abbreviations.

KLASNJA, Predrag; PRATT, Wanda. Healthcare in the pocket: Mapping the space of mobile-phone health interventions. Journal 
Aplicativos móveis de saúde para redução do comportamento sedentário

Deisy Terumi Ueno • Angélica Bonolo • Jean Augusto Coelho Guimarães • Ana Elisa Messetti Christofoletti • Priscila Missaki Nakamura • Eduardo Kokubun

of Biomedical Informatics, v. 45, n. 1, p. 184-198, 2012. DOI: 10.1016/j.jbi.2011.08.017. Disponível em: http://dx.doi.org/10.1016/j.jbi.2011.08.017.

KU, Po-Wen; STEPTOE, Andrew; LIAO, Yung; HSUEH, MingChun; CHEN, Li-Jung. A Threshold of Objectively-Assessed Daily Sedentary Time for All-cause Mortality in Older Adults: A MetaRegression of Prospective Cohort Studies. Journal of Clinical Medicine, v. 8, n. 4, p. 564, 2019. DOI: 10.3390/jcm8040564. LEVINE, James A. Non-exercise activity thermogenesis (NEAT). Nutrition Reviews, v. 286, n. 5, p. E675-E685, 2004. DOI: 10.1111/j.1753-4887.2004.tb00094.x.

LEWIS, Beth A.; NAPOLITANO, Melissa A.; BUMAN, Matthew P.; WILLIAMS, David M.; NIGG, Claudio R. Future directions in physical activity intervention research: expanding our focus to sedentary behaviors, technology, and dissemination. Journal of Behavioral Medicine, v. 40, n. 1, p. 112-126, 2017. DOI: 10.1007/s10865-0169797-8.

LITMAN, Leib; ROSEN, Zohn; SPIERER, David; WEINBERGERLITMAN, Sarah; GOLDSCHEIN, Akiva; ROBINSON, Jonathan. Mobile exercise apps and increased leisure time exercise activity: A moderated mediation analysis of the role of self-efficacy and barriers. Journal of Medical Internet Research, v. 17, n. 8, 2015. DOI: 10.2196/jmir.4142.

LUBANS, David R.; SMITH, Jordan J.; SKINNER, Geoff; MORGAN, Philip J. Development and implementation of a smartphone application to promote physical activity and reduce screen-time in adolescent boys. Frontiers in Public Health, v. 2, n. MAY, p. 1-11, 2014. DOI: 10.3389/fpubh.2014.00042.

LUPTON, Deborah. Critical Perspectives on Digital Health Technologies - Lupton - 2014 - Sociology Compass - Wiley Onl. Sociology Compass, v. 12, n. 2014, p. 1344-1359, 2014. Disponível em: https://onlinelibrary.wiley.com/doi/abs/10.1111/ soc4.12226. 
Aplicativos móveis de saúde para redução do comportamento sedentário

Deisy Terumi Ueno • Angélica Bonolo • Jean Augusto Coelho Guimarães • Ana Elisa Messetti Christofoletti • Priscila Missaki Nakamura • Eduardo Kokubun

MANINI, Todd M.; CARR, Lucas J.; KING, Abby C.; MARSHALL, Simon; ROBINSON, Thomas N.; REJESKI, W. Jack. Interventions to reduce sedentary behavior. Medicine and Science in Sports and Exercise, v. 47, n. 6, p. 1306-1310, 2015. DOI: 10.1249/ MSS.0000000000000519.

MAS, Adrià Muntaner; SAMPOL, Pere Palou; CONTI, Josep Vidal. Efectos de un programa de entrenamiento presencial vs prescripción a través de una aplicación móvil en personas mayores. Retos, v. 2041, n. 29, p. 32-37, 2016.

MCINTOSH, J. R. D.; JAY, S.; HADDEN, N.; WHITTAKER, P. J. Do E-health interventions improve physical activity in young people: a systematic review. Public Health, v. 148, p. 140-148, 2017. DOI: 10.1016/j.puhe.2017.04.001. Disponível em: http://dx.doi. org/10.1016/j.puhe.2017.04.001.

MIDDELWEERD, Anouk; MOLLEE, Julia S.; VAN DER WAL, C. N.; BRUG, Johannes; TE VELDE, Saskia J. Apps to promote physical activity among adults: A review and content analysis. International Journal of Behavioral Nutrition and Physical Activity, v. 11, n. 1, p. 1-9, 2014. DOI: 10.1186/s12966-014-0097-9.

OLIVEIRA, A. R. ..; ALENCAR, M. S. .. O uso de aplicativos de saúde para dispositivos móveis como fontes de informação e educação em saúde. RDBCl: Revista Digital de Biblioteconomia e Ciência da Informação, São Paulo, v. 15, n. 1, p. 234-245, 2017. DOI: 10.20396/rdbci.v0i0.8648137.

REZENDE, Leandro Fornias Machado; LOPES, Maurício Rodrigues; REY-LOṔEZ, Juan Pablo; MATSUDO, Victor Keihan Rodrigues; LUIZ, Olinda Do Carmo. Sedentary behavior and health outcomes: An overview of systematic reviews. PLoS ONE, v. 9, n. 8, 2014. DOI: 10.1371/journal.pone.0105620.

RHODES, Ryan E.; DEAN, Rachel N. Understanding physical inactivity: Prediction of four sedentary leisure behaviors. Leisure Sciences, v. 31, n. 2, p. 124-135, 2009. DOI: 10.1080/01490400802685948. 
Aplicativos móveis de saúde para redução do comportamento sedentário

Deisy Terumi Ueno • Angélica Bonolo • Jean Augusto Coelho Guimarães • Ana Elisa Messetti Christofoletti • Priscila Missaki Nakamura • Eduardo Kokubun

ROCHA, Thiago Augusto Hernandes; FACHINI, Luiz Augusto;

THUMÉ, Elaine; SILVA, Núbia Cristina Da; BARBOSA, Allan

Claudius Queiroz; CARMO, Maria Do; RODRIGUES, Júnia Marçal.

Saúde Móvel: novas perspectivas para a oferta de serviços em saúdeEpidemiologia e servicos de saude : revista do Sistema Unico de Saude do Brasil. Ministério da Saúde do Brasil, , 2016. DOI: 10.5123/s1679-49742016000100016. Disponível em: http:// www.iec.pa.gov.br/template_doi_ess.php?doi=10.5123/S167949742016000100016\&scielo=S2237-96222016000100159. Acesso em: 7 set. 2017.

SCHOEPPE, Stephanie; ALLEY, Stephanie; VAN LIPPEVELDE, Wendy; BRAY, Nicola A.; WILLIAMS, Susan L.; DUNCAN, Mitch J.; VANDELANOTTE, Corneel. Efficacy of interventions that use apps to improve diet, physical activity and sedentary behaviour: A systematic review. International Journal of Behavioral Nutrition and Physical Activity, v. 13, n. 1, 2016. DOI: 10.1186/s12966016-0454-y. Disponível em: http://dx.doi.org/10.1186/s12966-0160454-y.

TREMBLAY, Mark et al. Sedentary Behavior Research Network (SBRN) - Terminology Consensus Project process and outcome. International Journal of Behavioral Nutrition and Physical Activity, v. 14, n. 1, p. 1-17, 2017. DOI: 10.1186/s12966-017-05258.

\section{Publisher}

Universidade Federal de Goiás. Faculdade de Educação Física e Dança. Publicação no Portal de Periódicos UFG. As ideias expressadas neste artigo são de responsabilidade de seus autores, não representando, necessariamente, a opinião dos editores ou da universidade. 